\title{
Existence of Positive Solution for a Third-Order BVP with Advanced Arguments and Stieltjes Integral Boundary Conditions
}

\author{
Jian-Ping Sun, Ping Yan, Ya-Hong Zhao, and Fang-Di Kong \\ Department of Applied Mathematics, Lanzhou University of Technology, Lanzhou, Gansu 730050, China \\ Correspondence should be addressed to Jian-Ping Sun; jpsun2012@163.com
}

Received 20 June 2013; Revised 1 August 2013; Accepted 1 August 2013

Academic Editor: Fazal M. Mahomed

Copyright (C) 2013 Jian-Ping Sun et al. This is an open access article distributed under the Creative Commons Attribution License, which permits unrestricted use, distribution, and reproduction in any medium, provided the original work is properly cited.

A class of third-order boundary value problems with advanced arguments and Stieltjes integral boundary conditions is discussed. Some existence criteria of at least one positive solution are established. The main tool used is the Guo-Krasnoselskii fixed point theorem.

\section{Introduction}

Third-order differential equations arise in a variety of different areas of applied mathematics and physics, for example, in the deflection of a curved beam having a constant or varying cross-section, a three-layer beam, electromagnetic waves or gravity-driven flows, and so on [1].

Recently, third-order boundary value problems (BVPs for short) with integral boundary conditions, which cover thirdorder multipoint BVPs as special cases, have attracted much attention from many authors; see [2-6] and the references therein. In particular, in 2012, by using a fixed point theorem due to Avery and Peterson [7], Jankowski [4] established the existence of at least three nonnegative solutions to the following BVP:

$$
\begin{aligned}
& u^{\prime \prime \prime}(t)+h(t) f(t, u(\alpha(t)))=0, \quad t \in(0,1), \\
& u(0)=u^{\prime \prime}(0)=0, \quad u(1)=\beta u(\eta)+\lambda[u],
\end{aligned}
$$

where $\lambda$ denoted a linear functional on $C[0,1]$ given by

$$
\lambda[u]=\int_{0}^{1} u(t) d \Lambda(t)
$$

involving a Stieltjes integral with a suitable function $\Lambda$ of bounded variation. The measure $d \Lambda$ could be a signed one. The situation with a signed measure $d \Lambda$ was first discussed in $[8,9]$ for second-order differential equations; it was also discussed in $[10,11]$ for second-order impulsive differential equations. For some other related results, one can refer to [1214].

Among the boundary conditions in (1), only $u(1)$ is related to a Stieltjes integral. In this paper, we are concerned with the following third-order BVP with advanced arguments and Stieltjes integral boundary conditions:

$$
\begin{gathered}
u^{\prime \prime \prime}(t)+f(t, u(\alpha(t)))=0, \quad t \in(0,1), \\
u(0)=\gamma u(\eta)+\lambda[u], \quad u^{\prime \prime}(0)=0, \\
u(1)=\beta u(\eta)+\lambda[u] .
\end{gathered}
$$

Throughout this paper, we always assume that $\alpha:[0,1] \rightarrow$ $[0,1]$ is continuous and $\alpha(t) \geq t$ for $t \in[0,1], 0<\eta<1$, $0 \leq \gamma<\beta<1, \Lambda$ is a suitable function of bounded variation, and $\lambda[u]$ is defined as in (2). It is important to indicate that it is not assumed that $\lambda[u]$ is positive to all positive $u$. Some existence criteria of at least one positive solution to the BVP (3) are obtained by using the following well-known GuoKrasnoselskii fixed point theorem $[15,16]$.

Theorem 1. Let $E$ be a Banach space, and let $K$ be a cone in $E$. Assume that $\Omega_{1}$ and $\Omega_{2}$ are bounded open subsets of $E$ such that $0 \in \Omega_{1}, \bar{\Omega}_{1} \subset \Omega_{2}$, and let $T: K \cap\left(\bar{\Omega}_{2} \backslash \Omega_{1}\right) \rightarrow K$ be a completely continuous operator such that either

(1) $\|T u\| \leq\|u\|$ for $u \in K \cap \partial \Omega_{1}$ and $\|T u\| \geq\|u\|$ for $u \in K \cap \partial \Omega_{2}$ or 
(2) $\|T u\| \geq\|u\|$ for $u \in K \cap \partial \Omega_{1}$ and $\|T u\| \leq\|u\|$ for $u \in K \cap \partial \Omega_{2}$.

Then, $T$ has a fixed point in $K \cap\left(\bar{\Omega}_{2} \backslash \Omega_{1}\right)$.

\section{Preliminaries}

Let $\Delta=1-\gamma-(\beta-\gamma) \eta$. Then, $\Delta>0$.

Lemma 2. For any $y \in C[0,1]$, the $B V P$

$$
\begin{gathered}
u^{\prime \prime \prime}(t)=-y(t), \quad t \in(0,1), \\
u(0)=\gamma u(\eta)+\lambda[u], \quad u^{\prime \prime}(0)=0, \\
u(1)=\beta u(\eta)+\lambda[u]
\end{gathered}
$$

has the unique solution

$$
\begin{aligned}
u(t)= & \frac{1-(\beta-\gamma) \eta+(\beta-\gamma) t}{\Delta} \lambda[u] \\
& +\frac{\gamma+(\beta-\gamma) t}{\Delta} \int_{0}^{1} k(\eta, s) y(s) d s \\
& +\int_{0}^{1} k(t, s) y(s) d s, \quad t \in[0,1],
\end{aligned}
$$

where

$$
k(t, s)=\frac{1}{2} \begin{cases}(1-t)\left(t-s^{2}\right), & 0 \leq s \leq t \leq 1 \\ t(1-s)^{2}, & 0 \leq t \leq s \leq 1\end{cases}
$$

Proof. By integrating the differential equation in (4) three times from 0 to $t$ and using the boundary condition $u^{\prime \prime}(0)=$ 0 , we get

$$
u(t)=u(0)+u^{\prime}(0) t-\frac{1}{2} \int_{0}^{t}(t-s)^{2} y(s) d s, \quad t \in[0,1]
$$

So,

$$
u^{\prime}(0)=u(1)-u(0)+\frac{1}{2} \int_{0}^{1}(1-s)^{2} y(s) d s
$$

In view of (7), (8), and the boundary conditions $u(0)=$ $\gamma u(\eta)+\lambda[u]$ and $u(1)=\beta u(\eta)+\lambda[u]$, we have

$$
\begin{aligned}
u(t)= & {[\gamma+(\beta-\gamma) t] u(\eta)+\lambda[u] } \\
& +\int_{0}^{1} k(t, s) y(s) d s, \quad t \in[0,1]
\end{aligned}
$$

So,

$$
u(\eta)=\frac{1}{\Delta} \lambda[u]+\frac{1}{\Delta} \int_{0}^{1} k(\eta, s) y(s) d s
$$

Substituting (10) into (9), we get

$$
\begin{aligned}
u(t)= & \frac{1-(\beta-\gamma) \eta+(\beta-\gamma) t}{\Delta} \lambda[u] \\
& +\frac{\gamma+(\beta-\gamma) t}{\Delta} \int_{0}^{1} k(\eta, s) y(s) d s \\
& +\int_{0}^{1} k(t, s) y(s) d s, \quad t \in[0,1] .
\end{aligned}
$$

Lemma 3 (see [4]). Consider that $0 \leq k(t, s) \leq(1 / 2)(1+$ $s)(1-s)^{2},(t, s) \in[0,1] \times[0,1]$.

Throughout, we assume that the following conditions are fulfilled:

(C1) $f \in C([0,1] \times[0,+\infty),[0,+\infty))$,

(C2)

$$
\begin{gathered}
\int_{0}^{1} d \Lambda(t) \geq 0, \quad \int_{0}^{1} t d \Lambda(t) \geq 0, \\
\kappa(s)=\int_{0}^{1} k(t, s) d \Lambda(t) \geq 0, \quad s \in[0,1] .
\end{gathered}
$$

For convenience, we denote

$$
\begin{gathered}
\rho=[1-(\beta-\gamma) \eta] \int_{0}^{1} d \Lambda(t)+(\beta-\gamma) \int_{0}^{1} t d \Lambda(t), \\
\rho^{\prime}=\gamma \int_{0}^{1} d \Lambda(t)+(\beta-\gamma) \int_{0}^{1} t d \Lambda(t) .
\end{gathered}
$$

Obviously, $\rho, \rho^{\prime} \geq 0$. In the remainder of this paper, we always assume that $\rho<\Delta$.

Let $C[0,1]$ be equipped with the maximum norm. Then, $C[0,1]$ is a Banach space. Define

$$
K=\{u \in C[0,1]: u(t) \geq 0, t \in[0,1],
$$

$$
\left.\min _{t \in[\eta, 1]} u(t) \geq \Gamma\|u\|, \lambda[u] \geq 0\right\},
$$

where

$$
\Gamma=\min \left\{\frac{\beta(1-\eta)}{1-\beta \eta}, \frac{\beta \eta}{1-\gamma(1-\eta)}\right\} .
$$

Then, $K$ is a cone in $C[0,1]$.

Now, we define operators $T$ and $S$ on $K$ by

$$
\begin{array}{r}
(T u)(t)=\frac{1-(\beta-\gamma) \eta+(\beta-\gamma) t}{\Delta} \lambda[u]+(F u)(t), \\
t \in[0,1],
\end{array}
$$

$(S u)(t)=\frac{1-(\beta-\gamma) \eta+(\beta-\gamma) t}{\Delta-\rho} \lambda[F u]+(F u)(t)$,

$$
t \in[0,1]
$$


where

$$
\begin{aligned}
(F u)(t)= & \frac{\gamma+(\beta-\gamma) t}{\Delta} \int_{0}^{1} k(\eta, s) f(s, u(\alpha(s))) d s \\
& +\int_{0}^{1} k(t, s) f(s, u(\alpha(s))) d s, \quad t \in[0,1] .
\end{aligned}
$$

Lemma 4. Consider that $T, S: K \rightarrow K$.

Proof. Let $u \in K$. Then, it is easy to verify that

$$
(T u)^{\prime \prime}(t)=-\int_{0}^{t} f(s, u(\alpha(s))) d s \leq 0, \quad t \in[0,1],
$$

which shows that $T u$ is concave down on $[0,1]$. In view of

$$
\begin{aligned}
& (F u)(0)=\frac{\gamma}{\Delta} \int_{0}^{1} k(\eta, s) f(s, u(\alpha(s))) d s \geq 0, \\
& (F u)(1)=\frac{\beta}{\Delta} \int_{0}^{1} k(\eta, s) f(s, u(\alpha(s))) d s \geq 0,
\end{aligned}
$$

we have

$$
\begin{gathered}
(T u)(0)=\frac{1-(\beta-\gamma) \eta}{\Delta} \lambda[u]+(F u)(0) \geq 0, \\
(T u)(1)=\frac{1+(\beta-\gamma)(1-\eta)}{\Delta} \lambda[u]+(F u)(1) \geq 0 .
\end{gathered}
$$

So, $(T u)(t) \geq 0, t \in[0,1]$.

Now, we prove that $\min _{t \in[\eta, 1]}(T u)(t) \geq \Gamma\|T u\|$. To do it, we consider two cases.

Case 1. Let $(T u)(\eta) \leq(T u)(1)$. Then $\min _{t \in[\eta, 1]}(T u)(t)=$ $(T u)(\eta)$, and there exists $\bar{t} \in[\eta, 1]$ such that $\|T u\|=(T u)(\bar{t})$. Moreover,

$$
\frac{(T u)(\bar{t})-(T u)(0)}{\bar{t}-0} \leq \frac{(T u)(\eta)-(T u)(0)}{\eta-0} .
$$

So,

$$
\|T u\| \leq \frac{1}{\eta}(T u)(\eta)-\frac{1-\eta}{\eta}(T u)(0),
$$

which together with

$$
(T u)(0)=\gamma(T u)(\eta)+\lambda[u]
$$

implies that

$$
\|T u\| \leq \frac{1-\gamma(1-\eta)}{\eta}(T u)(\eta)
$$

that is,

$$
\min _{t \in[\eta, 1]}(T u)(t) \geq \frac{\eta}{1-\gamma(1-\eta)}\|T u\| .
$$

Case 2. Let $(T u)(\eta)>(T u)(1)$ and $\|T u\|=(T u)(\bar{t})$. Note that in this case $\min _{t \in[\eta, 1]}(T u)(t)=(T u)(1)$.
If $\bar{t} \in[0, \eta]$, then

$$
\frac{(T u)(1)-(T u)(\bar{t})}{1-\bar{t}} \geq \frac{(T u)(1)-(T u)(\eta)}{1-\eta} .
$$

So,

$$
\|T u\| \leq \frac{1}{1-\eta}(T u)(\eta)-\frac{\eta}{1-\eta}(T u)(1),
$$

which together with

$$
(T u)(\eta)=\frac{1}{\beta}((T u)(1)-\lambda[u])
$$

implies that

$$
\|T u\| \leq \frac{1-\beta \eta}{\beta(1-\eta)}(T u)(1) ;
$$

that is,

$$
\min _{t \in[\eta, 1]}(T u)(t) \geq \frac{\beta(1-\eta)}{1-\beta \eta}\|T u\| .
$$

If $\bar{t} \in(\eta, 1)$, then

$$
\frac{(T u)(\bar{t})-(T u)(\eta)}{\bar{t}-\eta} \leq \frac{(T u)(\eta)-(T u)(0)}{\eta-0} .
$$

So,

$$
\|T u\| \leq \frac{1}{\eta}(T u)(\eta)-\frac{1-\eta}{\eta}(T u)(0),
$$

which together with (23) and (28) implies that

$$
\|T u\| \leq \frac{1-\gamma(1-\eta)}{\beta \eta}(T u)(1) ;
$$

that is,

$$
\min _{t \in[\eta, 1]}(T u)(t) \geq \frac{\beta \eta}{1-\gamma(1-\eta)}\|T u\| .
$$

It follows from (25), (30), and (34) that

$$
\min _{t \in[\eta, 1]}(T u)(t) \geq \Gamma\|T u\| .
$$

Finally, we need to show that $\lambda[T u] \geq 0$. In view of

$$
\begin{aligned}
\lambda[F u]= & \int_{0}^{1} \frac{\gamma+(\beta-\gamma) t}{\Delta} \int_{0}^{1} k(\eta, s) f(s, u(\alpha(s))) d s d \Lambda(t) \\
& +\int_{0}^{1} \int_{0}^{1} k(t, s) f(s, u(\alpha(s))) d s d \Lambda(t) \\
= & \frac{\rho^{\prime}}{\Delta} \int_{0}^{1} k(\eta, s) f(s, u(\alpha(s))) d s \\
& +\int_{0}^{1} \kappa(s) f(s, u(\alpha(s))) d s \geq 0,
\end{aligned}
$$


we have

$$
\lambda[T u]=\frac{\rho}{\Delta} \lambda[u]+\lambda[F u] \geq 0 .
$$

This shows that $T: K \rightarrow K$. Similarly, we can prove that $S: K \rightarrow K$.

Lemma 5. The operators $T$ and $S$ have the same fixed points in $K$.

Proof. Suppose that $u \in K$ is a fixed point of $S$. Then,

$$
\begin{array}{r}
\lambda[u]=\int_{0}^{1}\left(\frac{1-(\beta-\gamma) \eta+(\beta-\gamma) t}{\Delta-\rho} \lambda[F u]\right. \\
+(F u)(t)) d \Lambda(t)=\frac{\Delta}{\Delta-\rho} \lambda[F u],
\end{array}
$$

which shows that

$$
\lambda[F u]=\frac{\Delta-\rho}{\Delta} \lambda[u]
$$

So,

$$
\begin{aligned}
u(t) & =(S u)(t) \\
& =\frac{1-(\beta-\gamma) \eta+(\beta-\gamma) t}{\Delta-\rho} \lambda[F u]+(F u)(t) \\
& =\frac{1-(\beta-\gamma) \eta+(\beta-\gamma) t}{\Delta} \lambda[u]+(F u)(t) \\
& =(T u)(t), \quad t \in[0,1],
\end{aligned}
$$

which indicates that $u$ is a fixed point of $T$.

Suppose that $u \in K$ is a fixed point of $T$. Then,

$$
\begin{array}{r}
\lambda[u]=\int_{0}^{1}\left(\frac{1-(\beta-\gamma) \eta+(\beta-\gamma) t}{\Delta} \lambda[u]\right. \\
+(F u)(t)) d \Lambda(t)=\frac{\rho}{\Delta} \lambda[u]+\lambda[F u],
\end{array}
$$

which shows that

$$
\lambda[u]=\frac{\Delta}{\Delta-\rho} \lambda[F u] .
$$

So,

$$
\begin{aligned}
u(t) & =(T u)(t) \\
& =\frac{1-(\beta-\gamma) \eta+(\beta-\gamma) t}{\Delta} \lambda[u]+(F u)(t) \\
& =\frac{1-(\beta-\gamma) \eta+(\beta-\gamma) t}{\Delta-\rho} \lambda[F u]+(F u)(t) \\
& =(S u)(t), \quad t \in[0,1],
\end{aligned}
$$

which indicates that $u$ is a fixed point of $S$.
Lemma 6. $T, S: K \rightarrow K$ is completely continuous.

Proof. First, by Lemma 4, we know that $T(K) \subset K$.

Next, we show that $T$ is compact. Let $D \subset K$ be a bounded set. Then, there exists $M_{1}>0$ such that $\|u\| \leq M_{1}$ for any $u \in D$. Since $\Lambda$ is a function of bounded variation, there exists $M_{2}>0$ such that $v_{\Delta^{\prime}}=\sum_{i=1}^{n}\left|\Lambda\left(t_{i}\right)-\Lambda\left(t_{i-1}\right)\right| \leq M_{2}$ for any partition $\Delta^{\prime}: 0=t_{0}<t_{1}<\cdots<t_{n-1}<t_{n}=1$. Let

$$
M_{3}=\sup \left\{f(t, u):(t, u) \in[0,1] \times\left[0, M_{1}\right]\right\} .
$$

Then, for any $u \in D$,

$$
\begin{aligned}
\|T u\|= & \max _{t \in[0,1]}(T u)(t) \\
\leq & \frac{1+(\beta-\gamma)(1-\eta)}{\Delta} \lambda[u] \\
& +\frac{\beta}{\Delta} \int_{0}^{1} k(\eta, s) f(s, u(\alpha(s))) d s \\
& +\frac{1}{2} \int_{0}^{1}(1+s)(1-s)^{2} f(s, u(\alpha(s))) d s \\
\leq & \frac{1+(\beta-\gamma)(1-\eta)}{\Delta} M_{1} M_{2} \\
& +\frac{\beta M_{3}}{\Delta} \int_{0}^{1} k(\eta, s) d s+\frac{5}{24} M_{3},
\end{aligned}
$$

which shows that $T(D)$ is uniformly bounded.

On the other hand, for any $\varepsilon>0$, since $k(t, s)$ is uniformly continuous on $[0,1] \times[0,1]$, there exists $\delta_{1}(\varepsilon)>0$ such that for any $t_{1}, t_{2} \in[0,1]$ with $\left|t_{1}-t_{2}\right|<\delta_{1}(\varepsilon)$,

$$
\left|k\left(t_{1}, s\right)-k\left(t_{2}, s\right)\right|<\frac{\varepsilon}{3 M_{3}}, \quad s \in[0,1] .
$$

Let $\delta=\min \left\{\delta_{1}(\varepsilon), \varepsilon \Delta / 3(\beta-\gamma) M_{1} M_{2}, \varepsilon \Delta / 3(\beta-\gamma) M_{3} \int_{0}^{1} k(\eta\right.$, $s) d s\}$. Then, for any $u \in D, t_{1}, t_{2} \in[0,1]$ with $\left|t_{1}-t_{2}\right|<\delta$, we have

$$
\begin{aligned}
\left|(T u)\left(t_{1}\right)-(T u)\left(t_{2}\right)\right| & \\
= & \mid \frac{(\beta-\gamma)\left(t_{1}-t_{2}\right)}{\Delta} \lambda[u]+\frac{(\beta-\gamma)\left(t_{1}-t_{2}\right)}{\Delta} \\
& \times \int_{0}^{1} k(\eta, s) f(s, u(\alpha(s))) d s \\
& +\int_{0}^{1}\left(k\left(t_{1}, s\right)-k\left(t_{2}, s\right)\right)
\end{aligned}
$$$$
\times f(s, u(\alpha(s))) d s \mid
$$ 


$$
\begin{aligned}
\leq & \frac{(\beta-\gamma)\left|t_{1}-t_{2}\right|}{\Delta} \lambda[u]+\frac{(\beta-\gamma)\left|t_{1}-t_{2}\right|}{\Delta} \\
& \times \int_{0}^{1} k(\eta, s) f(s, u(\alpha(s))) d s \\
& +\int_{0}^{1}\left|k\left(t_{1}, s\right)-k\left(t_{2}, s\right)\right| f(s, u(\alpha(s))) d s \\
\leq & \frac{(\beta-\gamma)\left|t_{1}-t_{2}\right| M_{1} M_{2}}{\Delta} \\
& +\frac{(\beta-\gamma)\left|t_{1}-t_{2}\right| M_{3}}{\Delta} \int_{0}^{1} k(\eta, s) d s \\
& +M_{3} \int_{0}^{1}\left|k\left(t_{1}, s\right)-k\left(t_{2}, s\right)\right| d s<\varepsilon,
\end{aligned}
$$

which shows that $T(D)$ is equicontinuous. It follows from the Arzela-Ascoli theorem that $T(D)$ is relatively compact. Thus, we have shown that $T$ is a compact operator.

Finally, we prove that $T$ is continuous. Suppose that $u_{n}$, $u \in K$ and $\lim _{n \rightarrow \infty} u_{n}=u$. Then, there exists $M_{4}>0$ such that $\|u\| \leq M_{4}$ and $\left\|u_{n}\right\| \leq M_{4}(n=1,2, \ldots)$. For any $\varepsilon>$ 0 , since $f(s, x)$ is uniformly continuous on $[0,1] \times\left[0, M_{4}\right]$, there exists $\delta>0$ such that for any $x_{1}, x_{2} \in\left[0, M_{4}\right]$ with $\left|x_{1}-x_{2}\right|<\delta$,

$$
\left|f\left(s, x_{1}\right)-f\left(s, x_{2}\right)\right|<\frac{\varepsilon}{(2 \beta / \Delta) \int_{0}^{1} k(\eta, s) d s+(5 / 12)},
$$$$
s \in[0,1] .
$$

At the same time, since $\lim _{n \rightarrow \infty} u_{n}=u$, there exists positive integer $N$ such that for any $n>N$,

$$
\left\|u_{n}-u\right\|<\min \left\{\delta, \frac{\varepsilon \Delta}{2[1+(\beta-\gamma)(1-\eta)]|\Lambda(1)-\Lambda(0)|}\right\} .
$$

It follows from (48) and (49) that for any $n>N$,

$$
\begin{aligned}
\left\|T u_{n}-T u\right\|= & \max _{t \in[0,1]}\left|\left(T u_{n}\right)(t)-(T u)(t)\right| \\
\leq & \frac{1+(\beta-\gamma)(1-\eta)}{\Delta}\left|\lambda\left[u_{n}\right]-\lambda[u]\right| \\
& +\frac{\beta}{\Delta} \int_{0}^{1} k(\eta, s) \mid f\left(s, u_{n}(\alpha(s))\right) \\
& -f(s, u(\alpha(s))) \mid d s
\end{aligned}
$$

$$
\begin{aligned}
& +\frac{1}{2} \int_{0}^{1}(1+s)(1-s)^{2} \mid f\left(s, u_{n}(\alpha(s))\right) \\
& -f(s, u(\alpha(s))) \mid d s \\
& \leq \frac{1+(\beta-\gamma)(1-\eta)}{\Delta}\left\|u_{n}-u\right\||\Lambda(1)-\Lambda(0)| \\
& +\int_{0}^{1}\left(\frac{\beta}{\Delta} k(\eta, s)+\frac{1}{2}(1+s)(1-s)^{2}\right) \\
& \quad \times\left|f\left(s, u_{n}(\alpha(s))\right)-f(s, u(\alpha(s)))\right| d s<\varepsilon,
\end{aligned}
$$

which indicates that $T$ is continuous. Therefore, $T: K \rightarrow$ $K$ is completely continuous. Similarly, we can prove that $S$ : $K \rightarrow K$ is also completely continuous.

\section{Main Results}

For convenience, we define

$$
\begin{gathered}
f^{0}=\limsup _{x \rightarrow 0^{+}} \max _{t \in[0,1]} \frac{f(t, x)}{x}, \quad f_{0}=\liminf _{x \rightarrow 0^{+}} \min _{t \in[\eta, 1]} \frac{f(t, x)}{x}, \\
f^{\infty}=\limsup _{x \rightarrow+\infty} \max _{t \in[0,1]} \frac{f(t, x)}{x}, \quad f_{\infty}=\liminf _{x \rightarrow+\infty} \min _{t \in[\eta, 1]} \frac{f(t, x)}{x}, \\
H_{1}=\frac{1+(\beta-\gamma)(1-\eta)}{\Delta-\rho}\left[\frac{\rho^{\prime}}{\Delta} \int_{0}^{1} k(\eta, s) d s+\int_{0}^{1} \kappa(s) d s\right] \\
+\frac{\beta}{\Delta} \int_{0}^{1} k(\eta, s) d s+\frac{5}{24}, \\
H_{2}=\Gamma\left\{\frac{1}{\Delta-\rho}\left[\frac{\rho^{\prime}}{\Delta} \int_{\eta}^{1} k(\eta, s) d s+\int_{\eta}^{1} \kappa(s) d s\right]\right. \\
\left.\quad+\frac{1}{\Delta} \int_{\eta}^{1} k(\eta, s) d s\right\} .
\end{gathered}
$$

Theorem 7. If $H_{1} f^{0}<1<H_{2} f_{\infty}$, then the BVP (3) has at least one positive solution.

Proof. Since $H_{1} f^{0}<1$, there exists $\varepsilon_{1}>0$ such that

$$
H_{1}\left(f^{0}+\varepsilon_{1}\right) \leq 1 \text {. }
$$

By the definition of $f^{0}$, we may choose $\rho_{1}>0$ so that

$$
f(t, x) \leq\left(f^{0}+\varepsilon_{1}\right) x, \quad t \in[0,1], x \in\left[0, \rho_{1}\right] .
$$

Let $\Omega_{1}=\left\{u \in C[0,1]:\|u\|<\rho_{1}\right\}$. Then, for any $u \in K \cap \partial \Omega_{1}$,

$$
0 \leq u(t) \leq\|u\|=\rho_{1}, \quad t \in[0,1],
$$


which together with (52) and (53) implies that

$$
(S u)(t)=\frac{1-(\beta-\gamma) \eta+(\beta-\gamma) t}{\Delta-\rho}
$$$$
\times\left[\frac{\rho^{\prime}}{\Delta} \int_{0}^{1} k(\eta, s) f(s, u(\alpha(s))) d s\right.
$$$$
\left.+\int_{0}^{1} \kappa(s) f(s, u(\alpha(s))) d s\right]
$$$$
+\frac{\gamma+(\beta-\gamma) t}{\Delta} \int_{0}^{1} k(\eta, s) f(s, u(\alpha(s))) d s
$$$$
+\int_{0}^{1} k(t, s) f(s, u(\alpha(s))) d s
$$$$
\leq \frac{1+(\beta-\gamma)(1-\eta)}{\Delta-\rho}
$$$$
\times\left[\frac{\rho^{\prime}}{\Delta} \int_{0}^{1} k(\eta, s) f(s, u(\alpha(s))) d s\right.
$$$$
\left.+\int_{0}^{1} \kappa(s) f(s, u(\alpha(s))) d s\right]
$$$$
+\frac{\beta}{\Delta} \int_{0}^{1} k(\eta, s) f(s, u(\alpha(s))) d s
$$$$
+\frac{1}{2} \int_{0}^{1}(1+s)(1-s)^{2} f(s, u(\alpha(s))) d s
$$$$
\leq\left(f^{0}+\varepsilon_{1}\right)\left\{\frac{1+(\beta-\gamma)(1-\eta)}{\Delta-\rho}\right.
$$$$
\times\left[\frac{\rho^{\prime}}{\Delta} \int_{0}^{1} k(\eta, s) u(\alpha(s)) d s\right.
$$$$
\left.+\int_{0}^{1} \kappa(s) u(\alpha(s)) d s\right]
$$$$
+\frac{\beta}{\Delta} \int_{0}^{1} k(\eta, s) u(\alpha(s)) d s
$$$$
\left.+\frac{1}{2} \int_{0}^{1}(1+s)(1-s)^{2} u(\alpha(s)) d s\right\}
$$$$
\leq\left(f^{0}+\varepsilon_{1}\right)\|u\|\left\{\frac{1+(\beta-\gamma)(1-\eta)}{\Delta-\rho}\right.
$$$$
\times\left[\frac{\rho^{\prime}}{\Delta} \int_{0}^{1} k(\eta, s) d s\right.
$$$$
\left.+\int_{0}^{1} \kappa(s) d s\right]
$$

$$
\begin{gathered}
\left.+\frac{\beta}{\Delta} \int_{0}^{1} k(\eta, s) d s+\frac{5}{24}\right\} \\
=H_{1}\left(f^{0}+\varepsilon_{1}\right)\|u\| \leq\|u\|, \quad t \in[0,1] .
\end{gathered}
$$

This shows that

$$
\|S u\| \leq\|u\|, \quad u \in K \cap \partial \Omega_{1} .
$$

On the other hand, since $1<H_{2} f_{\infty}$, there exists $\varepsilon_{2}>0$ such that

$$
H_{2}\left(f_{\infty}-\varepsilon_{2}\right) \geq 1
$$

By the definition of $f_{\infty}$, we may choose $\overline{\rho_{2}}>0$ so that

$$
f(t, x) \geq\left(f_{\infty}-\varepsilon_{2}\right) x, \quad t \in[\eta, 1], x \in\left[\overline{\rho_{2}},+\infty\right) .
$$

Let $\rho_{2}=\max \left\{2 \rho_{1}, \overline{\rho_{2}} / \Gamma\right\}$ and $\Omega_{2}=\left\{u \in C[0,1]:\|u\|<\rho_{2}\right\}$. Then, for any $u \in K \cap \partial \Omega_{2}$,

$$
u(t) \geq \Gamma\|u\|=\Gamma \rho_{2} \geq \overline{\rho_{2}}, \quad t \in[\eta, 1]
$$

which together with (57) and (58) implies that

$$
\begin{array}{r}
(\text { Su })(\eta)=\frac{1}{\Delta-\rho}\left[\frac{\rho^{\prime}}{\Delta} \int_{0}^{1} k(\eta, s) f(s, u(\alpha(s))) d s\right. \\
\left.+\int_{0}^{1} \kappa(s) f(s, u(\alpha(s))) d s\right] \\
+\frac{1}{\Delta} \int_{0}^{1} k(\eta, s) f(s, u(\alpha(s))) d s \\
\geq \frac{1}{\Delta-\rho}\left[\frac{\rho^{\prime}}{\Delta} \int_{\eta}^{1} k(\eta, s) f(s, u(\alpha(s))) d s\right. \\
\left.\quad+\int_{\eta}^{1} \kappa(s) f(s, u(\alpha(s))) d s\right] \\
+\frac{1}{\Delta} \int_{\eta}^{1} k(\eta, s) f(s, u(\alpha(s))) d s
\end{array}
$$




$$
\begin{aligned}
& \geq\left(f_{\infty}-\varepsilon_{2}\right)\left\{\frac { 1 } { \Delta - \rho } \left[\frac{\rho^{\prime}}{\Delta} \int_{\eta}^{1} k(\eta, s) u(\alpha(s)) d s\right.\right. \\
& \left.+\int_{\eta}^{1} \kappa(s) u(\alpha(s)) d s\right] \\
& \left.+\frac{1}{\Delta} \int_{\eta}^{1} k(\eta, s) u(\alpha(s)) d s\right\} \\
& \geq\left(f_{\infty}-\varepsilon_{2}\right) \Gamma\|u\|\left\{\frac { 1 } { \Delta - \rho } \left[\frac{\rho^{\prime}}{\Delta} \int_{\eta}^{1} k(\eta, s) d s\right.\right. \\
& \left.+\frac{1}{\Delta} \int_{\eta}^{1} k(\eta, s) d s\right\} \\
& =H_{2}\left(f_{\infty}-\varepsilon_{2}\right)\|u\| \geq\|u\| .
\end{aligned}
$$

This indicates that

$$
\|S u\| \geq\|u\|, \quad u \in K \cap \partial \Omega_{2} .
$$

Therefore, it follows from (56), (61), and Theorem 1 that the operator $S$ has one fixed point $u \in K \cap\left(\overline{\Omega_{2}} \backslash \Omega_{1}\right)$, which is a positive solution of the BVP (3).

Theorem 8. If $H_{1} f^{\infty}<1<H_{2} f_{0}$, then the BVP (3) has at least one positive solution.

Proof. Since $H_{2} f_{0}>1$, there exists $\varepsilon_{3}>0$ such that

$$
H_{2}\left(f_{0}-\varepsilon_{3}\right) \geq 1 \text {. }
$$

By the definition of $f_{0}$, we may choose $\rho_{3}>0$ so that

$$
f(t, x) \geq\left(f_{0}-\varepsilon_{3}\right) x, \quad t \in[\eta, 1], x \in\left[0, \rho_{3}\right] .
$$

Let $\Omega_{3}=\left\{u \in C[0,1]:\|u\|<\rho_{3}\right\}$. Then, for any $u \in K \cap \partial \Omega_{3}$,

$$
\Gamma\|u\| \leq u(t) \leq\|u\|=\rho_{3}, \quad t \in[\eta, 1]
$$

which together with (62) and (63) implies that

$$
\begin{aligned}
& (S u)(\eta)=\frac{1}{\Delta-\rho}\left[\frac{\rho^{\prime}}{\Delta} \int_{0}^{1} k(\eta, s) f(s, u(\alpha(s))) d s\right. \\
& \left.+\int_{0}^{1} \kappa(s) f(s, u(\alpha(s))) d s\right] \\
& +\frac{1}{\Delta} \int_{0}^{1} k(\eta, s) f(s, u(\alpha(s))) d s \\
& \geq \frac{1}{\Delta-\rho}\left[\frac{\rho^{\prime}}{\Delta} \int_{\eta}^{1} k(\eta, s) f(s, u(\alpha(s))) d s\right. \\
& \left.+\int_{\eta}^{1} \kappa(s) f(s, u(\alpha(s))) d s\right] \\
& +\frac{1}{\Delta} \int_{\eta}^{1} k(\eta, s) f(s, u(\alpha(s))) d s \\
& \geq\left(f_{0}-\varepsilon_{3}\right)\left\{\frac { 1 } { \Delta - \rho } \left[\frac{\rho^{\prime}}{\Delta} \int_{\eta}^{1} k(\eta, s) u(\alpha(s)) d s\right.\right. \\
& \left.+\int_{\eta}^{1} \kappa(s) u(\alpha(s)) d s\right] \\
& \left.+\frac{1}{\Delta} \int_{\eta}^{1} k(\eta, s) u(\alpha(s)) d s\right\} \\
& \geq\left(f_{0}-\varepsilon_{3}\right) \Gamma\|u\|\left\{\frac { 1 } { \Delta - \rho } \left[\frac{\rho^{\prime}}{\Delta} \int_{\eta}^{1} k(\eta, s) d s\right.\right. \\
& \left.+\int_{\eta}^{1} \kappa(s) d s\right] \\
& \left.+\frac{1}{\Delta} \int_{\eta}^{1} k(\eta, s) d s\right\} \\
& =H_{2}\left(f_{0}-\varepsilon_{3}\right)\|u\| \geq\|u\| \text {. }
\end{aligned}
$$

This shows that

$$
\|S u\| \geq\|u\|, \quad u \in K \cap \partial \Omega_{3} .
$$

On the other hand, since $H_{1} f^{\infty}<1$, there exists $\varepsilon_{4}>0$ so that

$$
H_{1}\left(f^{\infty}+\varepsilon_{4}\right)<1 .
$$

By the definition of $f^{\infty}$, we may choose $\overline{\rho_{4}}>0$ such that

$$
f(t, x) \leq\left(f^{\infty}+\varepsilon_{4}\right) x, \quad t \in[0,1], x \in\left[\overline{\rho_{4}},+\infty\right),
$$

which implies that

$$
f(t, x) \leq M+\left(f^{\infty}+\varepsilon_{4}\right) x, \quad t \in[0,1], x \in[0,+\infty),
$$

where

$$
M=\max \left\{f(t, x): t \in[0,1], x \in\left[0, \overline{\rho_{4}}\right]\right\} .
$$


Now, we choose $\rho_{4}=\max \left\{2 \rho_{3}, M H_{1} /\left(1-H_{1}\left(f^{\infty}+\varepsilon_{4}\right)\right)\right\}$, and let $\Omega_{4}=\left\{u \in C[0,1]:\|u\|<\rho_{4}\right\}$. Then, for any $u \in K \cap \partial \Omega_{4}$,

$$
0 \leq u(t) \leq\|u\|, \quad t \in[0,1],
$$

which together with (69) implies that

$$
\begin{aligned}
(S u)(t)= & \frac{1-(\beta-\gamma) \eta+(\beta-\gamma) t}{\Delta-\rho} \\
& \times\left[\frac{\rho^{\prime}}{\Delta} \int_{0}^{1} k(\eta, s) f(s, u(\alpha(s))) d s\right. \\
& \left.+\int_{0}^{1} \kappa(s) f(s, u(\alpha(s))) d s\right] \\
& +\frac{\gamma+(\beta-\gamma) t}{\Delta} \int_{0}^{1} k(\eta, s) f(s, u(\alpha(s))) d s \\
\leq & \left.\frac{1+(\beta-\gamma)(1-\eta)}{\Delta-\rho}, u(\alpha(s))\right) d s \\
& +\frac{1}{2} \int_{0}^{1}(1+s)(1-s)^{2} f(s, u(\alpha(s))) d s \\
& +\frac{\beta}{\Delta} \int_{0}^{1} k(\eta, s) f(s, u(\alpha(s))) d s \\
& \left.+\int_{0}^{1} \kappa(s) f(s, u(\alpha(s))) d s\right]
\end{aligned}
$$

This indicates that

$$
\|S u\| \leq\|u\|, \quad u \in K \cap \partial \Omega_{4} .
$$

Therefore, it follows from (66), (73), and Theorem 1 that the operator $S$ has one fixed point $u \in K \cap\left(\overline{\Omega_{4}} \backslash \Omega_{3}\right)$, which is a positive solution of the BVP (3).
Example 9. Consider the following BVP:

$$
\begin{aligned}
& u^{\prime \prime \prime}(t)+t\left[\frac{3359 u(\sqrt{t})}{913 e^{u(\sqrt{t})}}+\frac{5881(u(\sqrt{t}))^{2}}{17(u(\sqrt{t})+1)}\right]=0 \\
& t \in(0,1), \\
& u(0)=\frac{1}{4} u\left(\frac{1}{2}\right)+\int_{0}^{1} u(t)(2 t-1) d t, \quad u^{\prime \prime}(0)=0, \\
& u(1)=\frac{1}{2} u\left(\frac{1}{2}\right)+\int_{0}^{1} u(t)(2 t-1) d t .
\end{aligned}
$$

In view of $d \Lambda(t)=(2 t-1) d t$, we have

$$
\begin{array}{r}
\int_{0}^{1} d \Lambda(t)=0, \quad \int_{0}^{1} t d \Lambda(t)=\frac{1}{6}, \quad \kappa(s)=\frac{1}{12} s^{2}(1-s)^{2}, \\
s \in[0,1]
\end{array}
$$

At the same time, since $\gamma=1 / 4$ and $\beta=\eta=1 / 2$, a simple calculation shows that

$$
\begin{gathered}
\Delta=\frac{5}{8}, \quad \rho=\rho^{\prime}=\frac{1}{24}, \quad \Gamma=\frac{2}{7}, \\
\int_{0}^{1} k(\eta, s) d s=\frac{1}{16}, \quad \int_{\eta}^{1} k(\eta, s) d s=\frac{1}{96} \\
\int_{0}^{1} \kappa(s) d s=\frac{1}{360}, \quad \int_{\eta}^{1} \kappa(s) d s=\frac{1}{720} .
\end{gathered}
$$

So,

$$
H_{1}=\frac{913}{3360}, \quad H_{2}=\frac{17}{2940} .
$$

If we let $f(t, x)=t\left[\left(3359 x / 913 e^{x}\right)+\left(5881 x^{2} / 17(x+1)\right)\right]$, $(t, x) \in[0,1] \times[0,+\infty)$, then it is easy to compute that

$$
f^{0}=\frac{3359}{913}, \quad f_{\infty}=\frac{5881}{34},
$$

which together with (77) implies that

$$
H_{1} f^{0}=\frac{3359}{3360}<1<H_{2} f_{\infty}=\frac{5881}{5880} .
$$

Therefore, it follows from Theorem 7 that the BVP (74) has at least one positive solution.

\section{Acknowledgment}

This paper is supported by the Natural Science Foundation of Gansu Province of China (1208RJZA240). 


\section{References}

[1] M. Greguš, Third Order Linear Differential Equations, vol. 22, Reidel, Dordrecht, The Netherlands, 1987.

[2] J. R. Graef and J. R. L. Webb, "Third order boundary value problems with nonlocal boundary conditions," Nonlinear Analysis. Theory, Methods \& Applications A, vol. 71, no. 5-6, pp. 1542-1551, 2009.

[3] J. R. Graef and B. Yang, "Positive solutions of a third order nonlocal boundary value problem," Discrete and Continuous Dynamical Systems S, vol. 1, no. 1, pp. 89-97, 2008.

[4] T. Jankowski, "Existence of positive solutions to third order differential equations with advanced arguments and nonlocal boundary conditions," Nonlinear Analysis. Theory, Methods \& Applications A, vol. 75, no. 2, pp. 913-923, 2012.

[5] J.-P. Sun and H.-B. Li, "Monotone positive solution of nonlinear third-order BVP with integral boundary conditions," Boundary Value Problems, vol. 2010, Article ID 874959, 12 pages, 2010.

[6] Y. Wang and W. Ge, "Existence of solutions for a third order differential equation with integral boundary conditions," Computers \& Mathematics with Applications, vol. 53, no. 1, pp. 144154, 2007.

[7] R. I. Avery and A. C. Peterson, "Three positive fixed points of nonlinear operators on ordered Banach spaces," Computers \& Mathematics with Applications, vol. 42, no. 3-5, pp. 313-322, 2001.

[8] J. R. L. Webb and G. Infante, "Positive solutions of nonlocal boundary value problems: a unified approach," Journal of the London Mathematical Society, vol. 74, no. 3, pp. 673-693, 2006.

[9] J. R. L. Webb and G. Infante, "Positive solutions of nonlocal boundary value problems involving integral conditions," Nonlinear Differential Equations and Applications, vol. 15, no. 1-2, pp. 45-67, 2008.

[10] G. Infante, P. Pietramala, and M. Zima, "Positive solutions for a class of nonlocal impulsive BVPs via fixed point index," Topological Methods in Nonlinear Analysis, vol. 36, no. 2, pp. 263-284, 2010.

[11] T. Jankowski, "Positive solutions for second order impulsive differential equations involving Stieltjes integral conditions," Nonlinear Analysis. Theory, Methods \& Applications A, vol. 74, no. 11, pp. 3775-3785, 2011.

[12] C. Bandle, "An eigenvalue problem with mixed boundary conditions and trace theorems," Banach Journal of Mathematical Analysis, vol. 2, no. 2, pp. 68-75, 2008.

[13] D. B. Pachpatte, "Properties of some dynamic integral equation on time scales," Annals of Functional Analysis, vol. 4, no. 2, pp. 12-26, 2013.

[14] N. Shayanfar, M. Hadizadeh, and A. Amiraslani, "Integral operators acting as variables of the matrix polynomial: application to system of integral equations," Annals of Functional Analysis, vol. 3, no. 2, pp. 170-182, 2012.

[15] D. J. Guo and V. Lakshmikantham, Nonlinear Problems in Abstract Cones, vol. 5, Academic Press, Boston, Mass, USA, 1988.

[16] M. A. Krasnoselskii, Positive Solutions of Operator Equations, Noordhoff, Groningen, The Netherlands, 1964. 


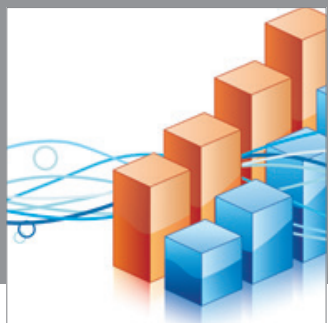

Advances in

Operations Research

mansans

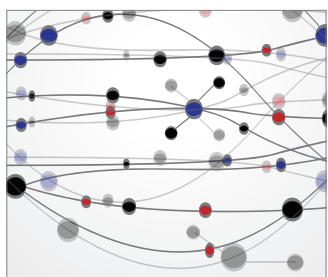

The Scientific World Journal
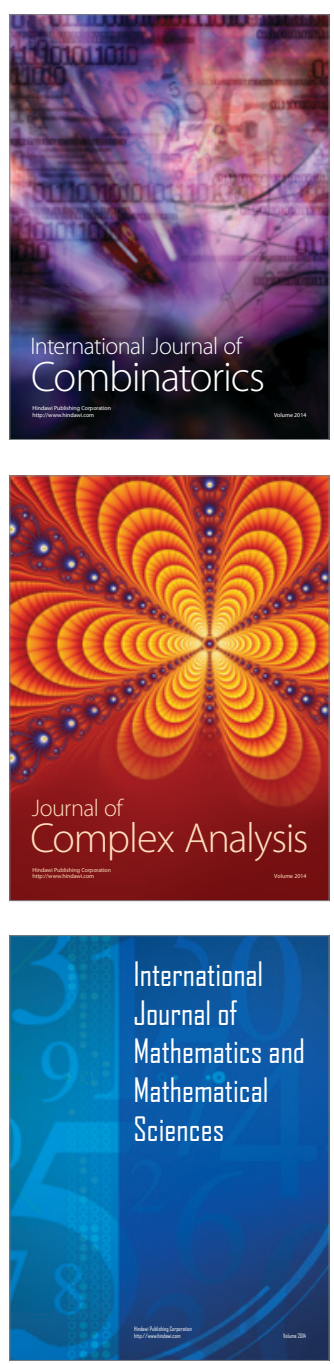
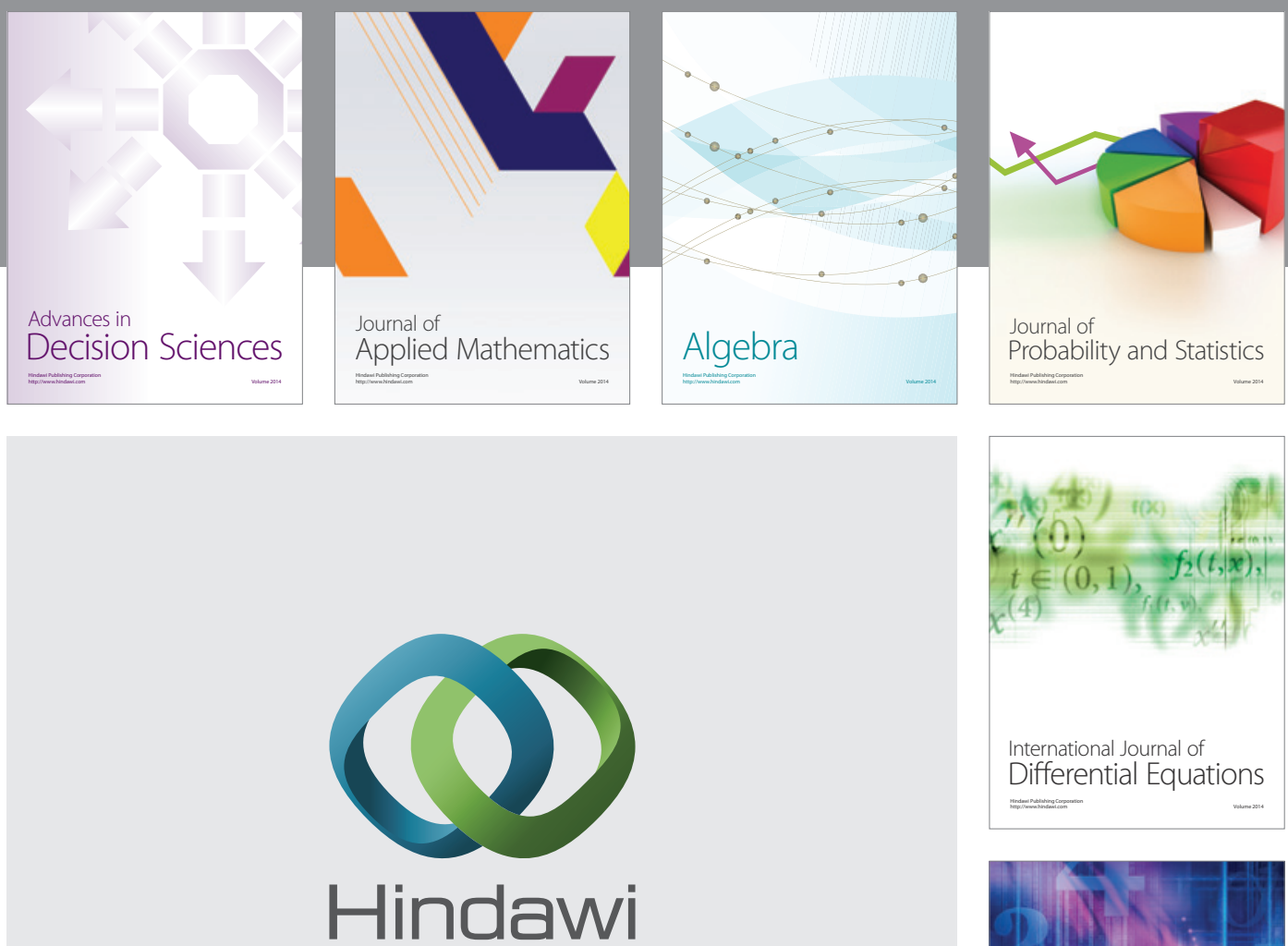

Submit your manuscripts at http://www.hindawi.com
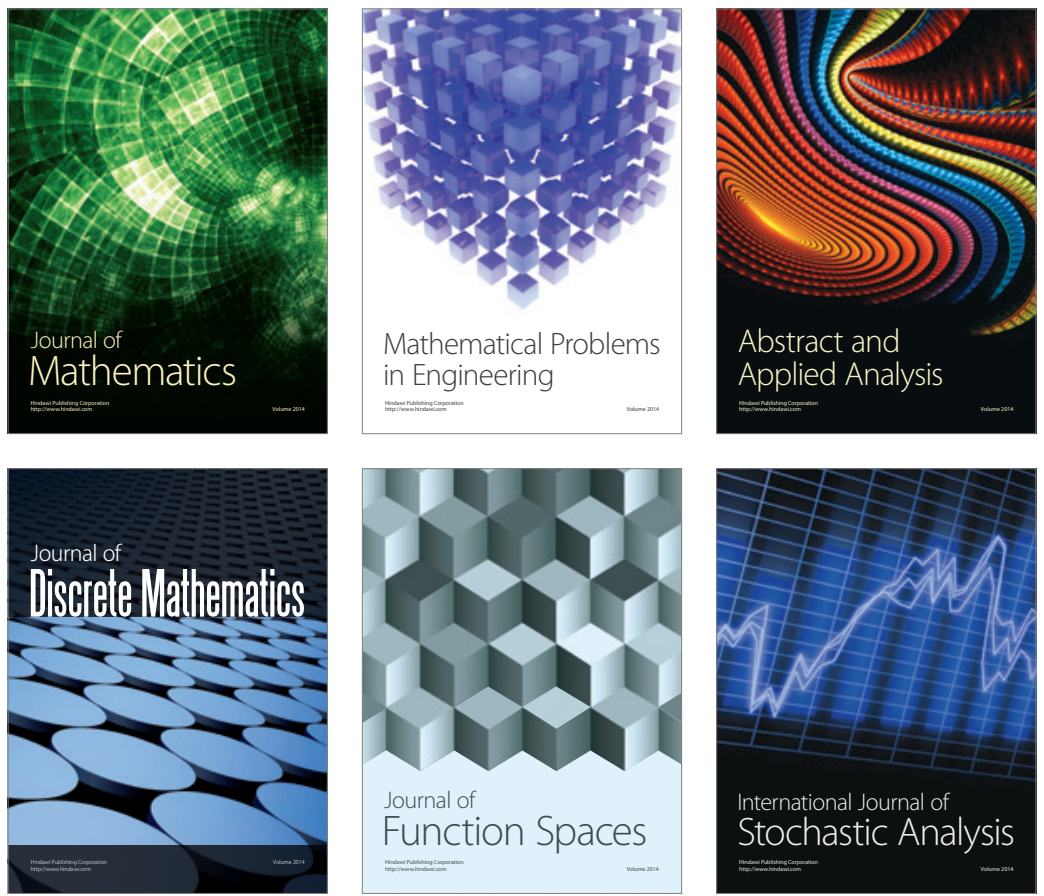

Journal of

Function Spaces

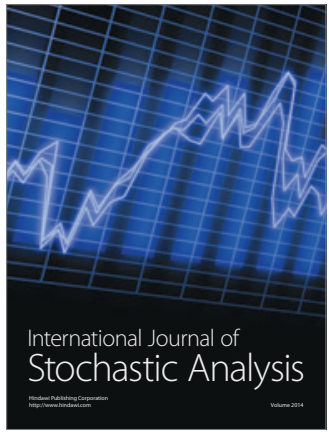

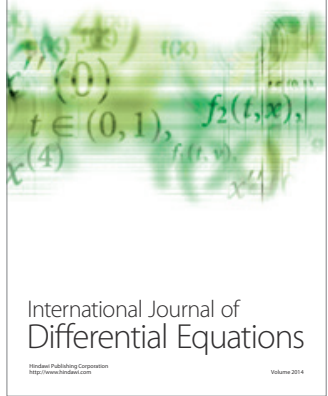
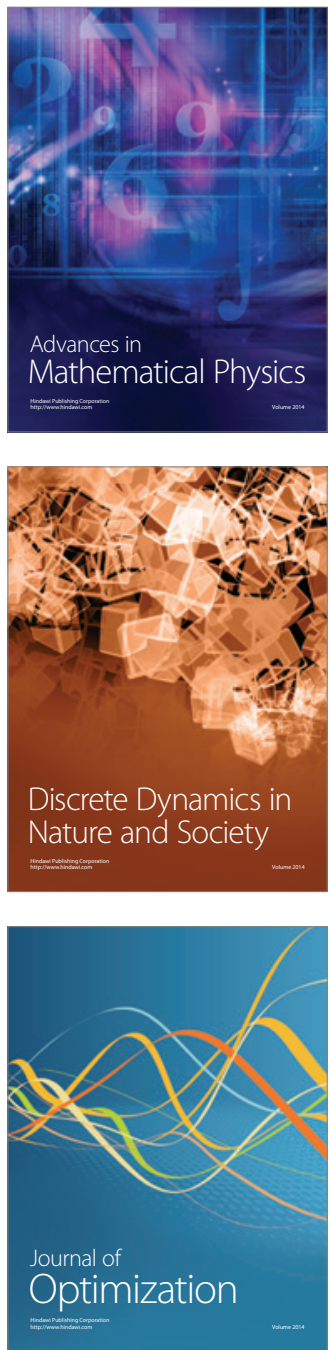